\title{
DUAL-PULSE LASER-INDUCED BREAKDOWN SPECTROMETRY OF BRONZE ALLOYS AND COATINGS
}

\author{
K. F. Ermalitskaia, ${ }^{*}$ Y. S. Voropay, and A. P. Zajogin
}

UDC 533.9.082;533.924;621.373.8

The possibilities of using dual-pulse laser atomic-emission spectroscopy for both the estimation of elemental concentrations in bronzes and layer-by-layer analysis of a bronze coating on steel bead wire have been examined. Two methods have been proposed to lower the radiation flux density $q$ in studies of thin metallic layers. These were based respectively on using optical filters and defocusing the radiation with respect to a sample surface. It has been found that the decrease of $q$ results in reducing the influence of tin on copper evaporation under dual-pulse laser irradiation.

Key words: laser ablation, dual laser pulses, laser plasma, laser atomic-emission spectroscopy, layer-by-layer quantitative analysis, bronzes, bronze coating.

Introduction. Laser-induced breakdown spectroscopy (LIBS) is a method of qualitative and quantitative analysis that is based on recording emission spectra of laser-induced plasma. It depends fundamentally on the interaction of powerful optical radiation with a sample surface that produces heat, melting, vaporization, and atomization of a substance and formation of ablation plasma. One of the most rapidly developing areas of LIBS that can increase significantly the intensity of the spectral lines and, therefore, the analytical sensitivity is the use of dual laser pulses (DLP) that are phase-shifted relative to each other as the excitation source for the spectra [1,2]. The principal reason for the increase of optical emission is the formation of a region near the surface with increased temperature and particle density. Furthermore, heating of the sample by the first pulse and the additional excitation of atoms in the initial plasma by the second pulse are also responsible for the strengthening of the analytical signal.

The advantages of LIBS are the highly localized sampling area; the small amounts of substance vaporized by the radiation; the weak dependence of the ablation process on the sample physicochemical properties; and ability to use untreated surfaces. Because of these advantages, LIBS is used to analyze various objects such as industrial raw materials and finished products, minerals and soils, and biological objects [3-5].

LIBS of bronzes has been the subject of several theoretical and experimental studies [6-8]. However, the attention in most of these was focused on quantitative analysis of works of art [6] or objects located under water [7]. Yet bronzes are rather common construction materials that are used to fabricate massive items and as coatings on steel wire in order to protect it and improve the adhesion of the surface to resin. LIBS studies of thin metal layers involved primarily single-component coatings such as galvanized iron [9] and copper deposited on steel boiler pipes [10]. The requirements imposed on the excitation source of the spectrum are different for analyzing works of art and industrial products made of bronze. In the first instance, it is very important to minimize surface damage by the laser radiation; in the second, to minimize the determination error of element concentrations to the corresponding GOST level. This is usually achieved by using multiple surface excitations.

The goal of our work was to study the processes by which bronze components enter the plume, to determine the optimal DLP parameters in order to minimize uncertainties in quantitative analysis of the compositions of bronzes in both massive items and micron-thin coatings, and to develop a quantitative analytical method for micron-thin metal coatings.

\footnotetext{
*To whom correspondence should be addressed.
}

Belarusian State University, 4 Nezavisimosti Ave., Minsk, 220030, Belarus; e-mail: ermalitskaia@gmail.com. Translated from Zhurnal Prikladnoi Spektroskopii, Vol. 77, No. 2, pp. 165-172, March-April, 2010. Original article submitted June 30, 2009. 
TABLE 1. Average Content (\%) of Elements in Standard Bronze Samples

\begin{tabular}{||c|c|c|c|c|c|c||}
\hline \hline Sample No. & Sn & Zn & P & Pb & Sb & $\mathrm{Cu}$ \\
\hline 111 & 7.94 & 0.12 & 0.224 & 0.024 & 0.108 & 91.45 \\
112 & 8.90 & 0.21 & 0.460 & 0.050 & 0.200 & 89.74 \\
113 & 9.90 & 0.31 & 0.750 & 0.079 & 0.310 & 88.16 \\
114 & 10.70 & 0.75 & 1.070 & 0.290 & 0.770 & 85.29 \\
115 & 11.80 & 0.42 & 1.470 & 0.151 & 0.490 & 85.29 \\
\hline
\end{tabular}

Experimental. The studies were carried out on an LSS-1 laser spectrometer produced by the Belarusian-Japanese joint venture LOTIS-TII (Minsk). Radiation from a dual-pulse Nd:YAG-laser with active gain modulation was used as the source for vaporizing the sample and exciting the atomic spectra. The principal parameters of the laser radiation were wavelength $1064 \mathrm{~nm}$, pulse repetition frequency $10 \mathrm{~Hz}$, duration at half-height $15 \mathrm{nsec}$, pulse energy $E_{\mathrm{pul}}=10-60 \mathrm{~mJ}$, time interval between DLP $\Delta t=0-100 \mu \mathrm{sec}(1 \mu \mathrm{sec}$ step). The energies of both pulses were the same with fixed pumping energy and interval $\Delta t$ [11]. The null interpulse interval corresponded to simultaneous shots of both laser pulses on the surface. This could be considered a single pulse, the power of which was equal to the total power of the DLP. The leading front of the plasma formed by the first laser pulse emitted an intense continuous spectrum. The analytical signal was recorded synchronously with the arrival of the second pulse in order to eliminate any effect of that spectrum on the intensity of the sample emission lines. The continuum emitted by the leading front of the plasma formed by the second pulse was much weaker because of the reduced particle density of the surrounding atmosphere in the region near the surface.

Radiation of the sample plasma was fed using two achromatic objectives into the entrance end of a dual light-guide and directed into two SDH-1 compact polychromators (SOLAR Laser Systems, Minsk). A diffraction grating of 1200 lines $/ \mathrm{mm}$ was used in the SDH-1. For this, the average inverse linear dispersion was $5.3 \mathrm{~nm} / \mathrm{mm}$; spectral resolution, $0.12 \mathrm{~nm}$. The working range of the first polychromator was 190-340 nm; of the second, 265-800 nm (recorded spectral width in both instances $\sim 150 \mathrm{~nm}$, range of the second polychromator retuned by a microscrew). The SDH-1 polychromator is a diffraction spectrograph that provides a linear spectral scan for a recording system based on a 3648-pixel multi-channel TCD1304AP detector (Toshiba). The LSS-1 laser spectrometer was connected to a computer using a USB cable. All experiments were performed in air at normal atmospheric pressure. The sample remained motionless during the laser ablation.

Standard bronze samples from set M85 (SSS 2659-83-2663-83) (RF State Research and Design Institute of Alloys and Non-Ferrous Metal Processing, 1998) were used to study processes of entry of alloy material into the plume and to develop quantitative analytical procedures. Table 1 lists the contents of principal elements in the standard samples.

The second main task of the study was to develop quantitative analytical procedures for micron-thin bronze metal coatings. Samples of bronze-coated steel bead wire that is used to manufacture tires (RUP Belarusian Metallurgical Plant, Zhlobin) were studied. The sample diameter was $1.5 \mathrm{~mm}$; coating thickness, $1.5 \mu \mathrm{m}$. The bronze coating was applied galvanically on steel wire by simultaneous deposition of $\mathrm{Cu}$ and $\mathrm{Sn}$ without heating. Layer-by-layer analysis requires that a constant layer thickness be removed by each pair of DLP. The crater depth on the sample surface and the layer thickness were determined using a Linnik MII-4 microinterferometer.

The amount of vaporized substance was determined from the radiation flux density $q$ on the sample surface:

$$
q \approx E_{\mathrm{pul}} / \tau S,
$$

where $E_{\text {pul }}$ and $\tau$ are the energy and duration of the pulse at half-height and $S$ is the effective area of the laser spot on the target. For $E_{\text {pul }}=60 \mathrm{~mJ}$ and $q \sim 10^{10} \mathrm{~W} / \mathrm{cm}^{2}$, the average crater depth on the sample surface after the DLP shot was $5 \mu \mathrm{m}$. The number of vaporized layers for performing the layer-by-layer analysis of the bronze coating should be $\geq 10$ with thickness $d \leq 0.15 \mu \mathrm{m}$, which predetermines the need to decrease the radiation flux density on the sample surface. Two methods were proposed for reducing $q$. These were use of light filters (optical glasses) with dif- 


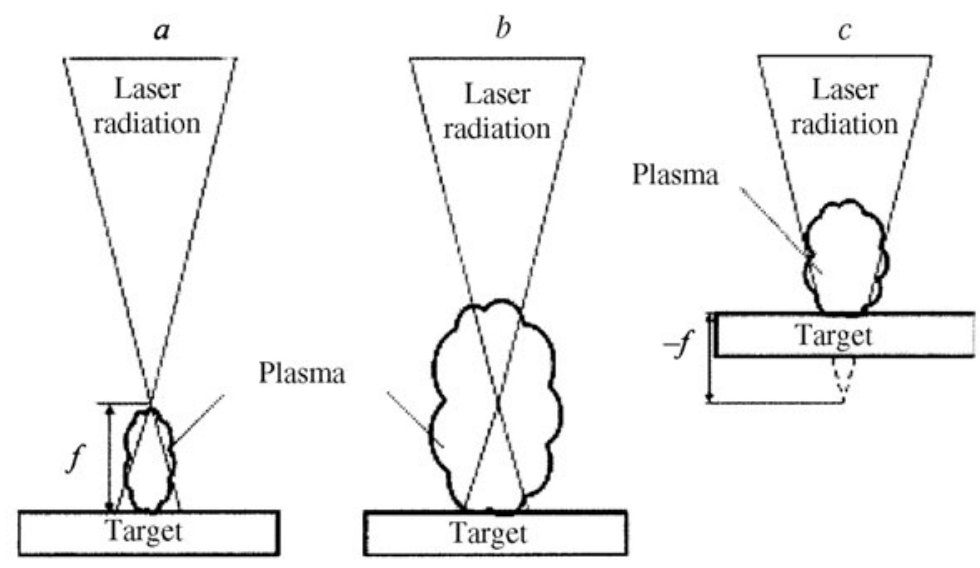

Fig. 1. Diagram of positive (a, $\Delta t<4 \mu \mathrm{sec} ; \mathrm{b}, \Delta t>4 \mu \mathrm{sec}$ ) and negative (c) defocusing of laser radiation relative to sample surface.

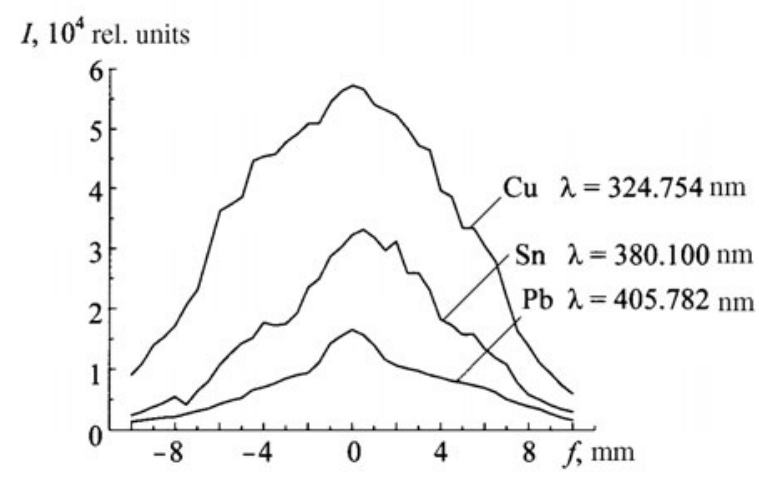

Fig. 2. Intensity of $\mathrm{Cu}, \mathrm{Sn}$, and $\mathrm{Pb}$ spectral lines as functions of defocusing parameter $f$.

ferent transmission coefficients for the laser radiation and defocusing of the beam relative to the sample surface with an increased area of the laser spot. The amount of vaporized substance decreased with the use of light filters because of a decrease of $q$. As a result, the intensity of the $\mathrm{Sn}, \mathrm{Zn}$, and $\mathrm{Pb}$ spectral lines was at the background level for the required thickness of the analyzed layer. This prevented this method for decreasing $q$ from being used for analytical purposes.

A different picture was observed for beam defocusing. More substance than when light filters were used entered the plasma for the same $q$ because of the increased area of the laser spot. The distance $f$ from the focal point to the sample (defocusing parameter) was set by shifting the sample stage with the sample fixed to it along the optical axis. Two types of defocusing, positive and negative, were possible depending on the position of the focal point relative to the surface (Fig. 1).

A study of the intensity $I$ of the spectral lines as a function of the defocusing parameter showed that $I$ depended on the amplitude of $f$ and not on its sign. Figure 2 shows the intensity of the strongest spectral lines of $\mathrm{Cu}$, $\mathrm{Sn}$, and $\mathrm{Pb}$ as functions of the defocusing parameter.

With negative defocusing, almost the whole plasma volume expanded within the cone of the laser radiation (Fig. 1c). For $f>0$, the laser beam was focused in the plasma volume. The density of vaporized substance near the surface was high for small interpulse intervals before the arrival of the second pulse (Fig. 1a). The energy of the second pulse was dissipated by the additional excitation of atoms in the plasma, because of which the extent of surface 
destruction and, therefore, the thickness of the removed layer decreased. This caused the conditions for exciting the atomic spectra to become unstable and, as a result, the experimental uncertainty of the quantitative analysis to rise. Increasing the interpulse interval resulted in a significant part of the substance vaporized by the first pulse to be located outside the effective zone of the second pulse, i.e., it was not additionally excited (Fig. 1b). Defocusing the laser beam during layer-by-layer analysis made it possible to increase the intensities of the element spectral lines and the analytical possibilities of the method. However, the probability of a breakdown in air by the first laser pulse, so-called laser sparking [12], existed for $f>0$. This could change the fraction of laser energy that reached the target.

Results and Discussion. The main problem with spectral analysis of bronzes involved matrix effects that increased the analytical uncertainty. Several studies on LIBS of bronze alloys recommended the use of calibration-free approaches [5, 13]. However, intermediate calculations need to be minimized for measurements made on industrial items. Therefore, we faced the problem of developing quantitative analysis procedures that were based on the construction of calibration curves.

Such quantitative analytical procedures for bronzes are based on the use of intensity $I$ of element spectral lines as a function of their concentration in the alloy $C$ :

$$
I=f(C) .
$$

The total intensity of an element spectral line that was accumulated over the total number of DLP was used as the analytical signal. The intensity of spectral lines for the principal bronze components was analyzed during the experiment for multiple DLP shots with different parameters. The strongest spectral lines for $\mathrm{Zn}, \mathrm{Pb}$, and $\mathrm{Sn}(\mathrm{Zn}, \lambda=$ $334.502 \mathrm{~nm} ; \mathrm{Pb}, \lambda=405.782$; $\mathrm{Sn}, \lambda=380.100$ ) were selected as the analytical lines in order to decrease the uncertainty in the element concentrations. Because of the high $\mathrm{Cu}$ content in the bronze, use of the strong $\mathrm{Cu}$ resonance lines (324.754 and $327.396 \mathrm{~nm}$ ) led to a self-absorption effect, i.e., multiple absorption and re-emission of light by atoms of the same element. Hence, the greater the $\mathrm{Cu}$ content in the plasma was, the more extensive the self-absorption process would be. This was equivalent to increasing the lifetime of the atom in the excited state and, as a result, decreasing the probability of spontaneous emission of a light quantum at a given wavelength $\lambda$. The self-absorption effect resulted ultimately in the intensity of the element spectral line decreasing as its concentration in the sample increased (formula obtained by Lomakin and Shaibe) [14]:

$$
I=a C^{b},
$$

where $b=f(C)$ is a decaying function of the element concentration $C$. We used the strong non-resonant $\mathrm{Cu}$ line with $\lambda=330.795 \mathrm{~nm}$ in our studies.

A previous study of dual-pulse LIBS of $\mathrm{Cu}$ alloys [15] showed that calibration curves that are constructed in standard linear coordinates $I=k_{1} C+k_{2}$ cannot be approximated by a straight line for all elements with the exception of $\mathrm{Zn}$. The researchers recommended using relative coordinates:

$$
\frac{I_{i}}{I_{\mathrm{Zn}}}=k_{3} \frac{C_{i}}{C_{\mathrm{Zn}}}+k_{4}
$$

where $I_{i}, I_{\mathrm{Zn}}$ and $C_{i}, C_{\mathrm{Zn}}$ are intensities of spectral lines and concentrations of the determined element and $\mathrm{Zn}$ in the sample. The previous study [15] developed an analytical procedure for use with samples in which the concentrations of principal elements varied over wide ranges: $\mathrm{Cu}$, from 62 to $90 \% ; \mathrm{Zn}, 0-32 ; \mathrm{Sn}, 0.5-13$. However, a sample with $62 \% \mathrm{Cu}$ and $32 \% \mathrm{Zn}$ is a bronze and in this instance the mutual effect of both principal components on entering the plasma must be taken into account.

An experiment showed that the calibration curves in standard coordinates for $\mathrm{Zn}, \mathrm{Pb}$, and $\mathrm{Sn}$ were linear for any laser-radiation parameters (pulse energy, interpulse interval, number of pulses) over the concentration range of the elements in the samples (Table 1). The intensities of the spectral lines of metals in the bronzes were strongest for an interpulse interval of $10-12 \mu \mathrm{sec}$ at the highest $(60 \mathrm{~mJ})$ energy of each DLP [16]. The direct proportionality of the line intensity to $E_{\text {pul }}$ was related to the increased amount of vaporized substance as the pulse energy increased. The calibration curves for $\mathrm{Cu}$ that were constructed in standard coordinates could not be used for analytical purposes. 

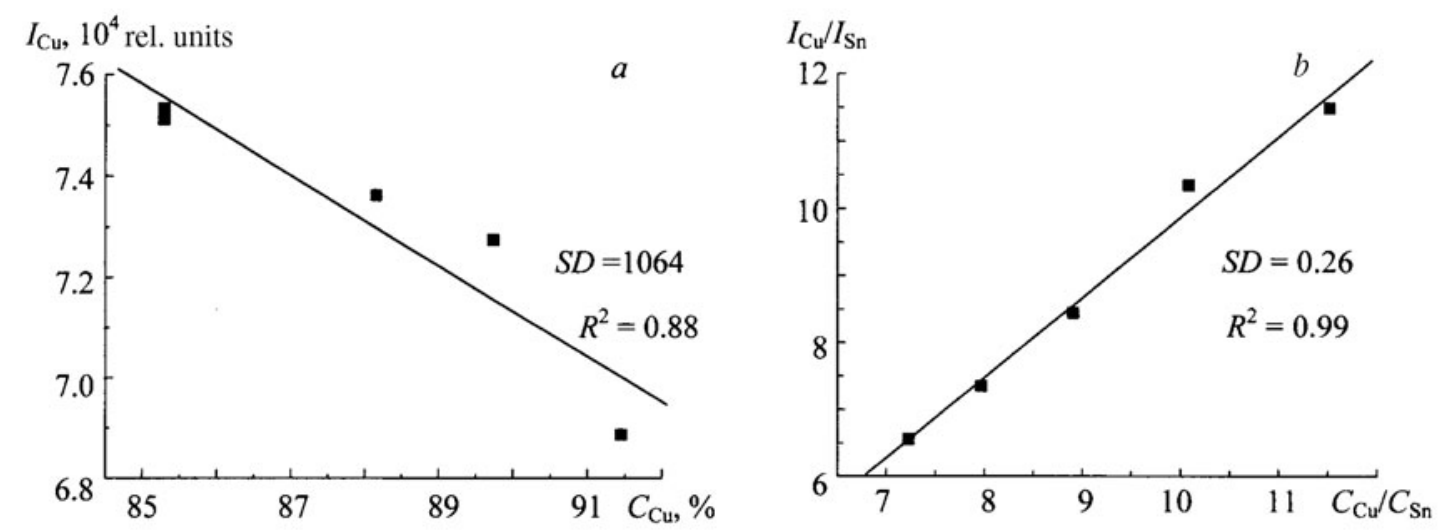

Fig. 3. Calibration curves in standard (a) and relative (b) coordinates for determining $\mathrm{Cu}$ concentration in bronzes by dual-pulse laser-induced breakdown spectroscopy.

Thus, the experimental points for $E_{\text {pul }}=20 \mathrm{~mJ}$ could be approximated by a straight line with positive slope $\left(R^{2}=\right.$ 0.7). Performing quantitative analysis of bronze alloys at pulse energy $20 \mathrm{~mJ}$ is unfeasible because of the low correlation coefficient of the linear approximation of the experimental points and the low spectral line intensities of the other bronze components. For $E_{\text {pul }}=40 \mathrm{~mJ}$, the function $I(C)$ is non-linear; for $60 \mathrm{~mJ}$, the calibration curve has a negative slope. Relative coordinates must be used to determine the $\mathrm{Cu}$ concentration in the bronzes:

$$
\frac{I_{\mathrm{Cu}}}{I_{\mathrm{Sn}}}=k \frac{C_{\mathrm{Cu}}}{C_{\mathrm{Sn}}}+m,
$$

where $I_{\mathrm{Cu}}, I_{\mathrm{Sn}}$ and $C_{\mathrm{Cu}}, C_{\mathrm{Sn}}$ are the spectral line intensities for $\mathrm{Cu}$ and $\mathrm{Sn}$ and their concentrations in the bronzes; $k$ and $m$, coefficients. Figure 3 shows calibration curves for determining $\mathrm{Cu}$ concentrations in bronzes that were constructed in standard and relative coordinates. The squares of the correlation coefficient $R^{2}$ and the mean-square deviations $S D$ are also given and indicate that the linear approximation of the experimental points can be used. The results indicated that $\mathrm{Sn}$ in the bronzes affected entry of $\mathrm{Cu}$ into the plume and that this effect was greater as the energy of the laser pulse increased. This was seen in the deviation from linearity of the calibration curves in standard coordinates. The experimentally determined optimal laser-radiation parameters for conducting quantitative analysis of bronzes that produced the strongest spectral lines of all components were $E_{\mathrm{pul}}=60 \mathrm{~mJ}$ and $\Delta t=10 \mu \mathrm{sec}$.

The development of an analytical procedure for layer-by-layer quantitative analysis of micron-thin bronze coatings of steel wires must take into account that vaporization processes of the substance and, consequently, the parameters of the plasma itself will vary because of the reduced radiation flux density [17]. It was found experimentally that the defocusing parameter $f$ should be $\pm 10 \mathrm{~mm}$ and the flux density $\sim 2.5 \cdot 10^{7} \mathrm{~W} / \mathrm{cm}^{2}$ upon reaching a vaporized layer thickness of $0.1 \mu \mathrm{m}$ using an objective with focal length $100 \mathrm{~mm}$. Primarily individual atoms enter the plume for this value of $q$ upon vaporization of the bronze coating by defocused laser radiation [18]. The issue of the target material was determined by the alloy thickness on the sample surface, which depended on the surface temperature $T$ [19]:

$$
T=\frac{2 q(1-R) \sqrt{a \tau}}{k \sqrt{\pi}}+T_{\mathrm{i}},
$$

where $R$ is the surface reflectance coefficient; $T_{\mathrm{i}}$, the initial temperature (equal to ambient for the first pulse of the DLP); $\tau$, pulse length; $k$, thermal conductivity; $a$, temperature conductivity of the sample. 


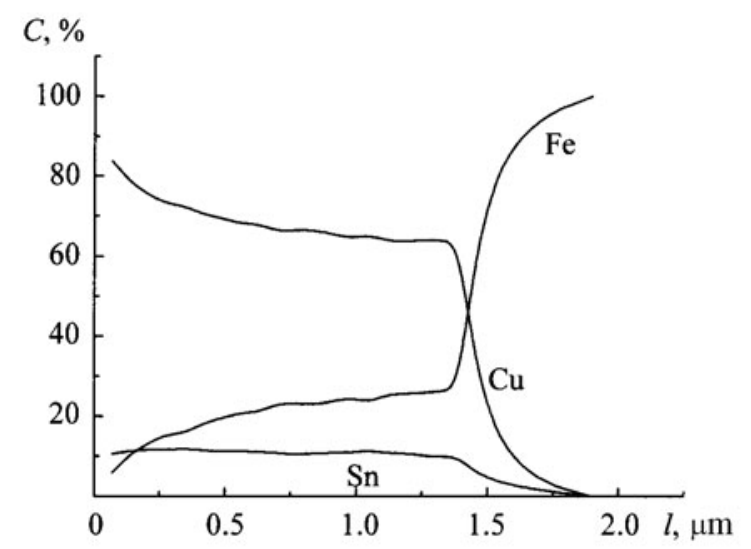

Fig. 4. Concentration distribution of elements with depth 1 of bronze coating of steel bead wire.

The decreased power density resulted in a significant reduction of the surface temperature compared with focused radiation. The thickness of the fused layer $x_{\mathrm{f}}$ on the bronze surface can be estimated using the formula [18]:

$$
x_{\mathrm{f}}=\frac{k \lambda_{\mathrm{f}}}{q(1-R) C} \ln \frac{\left(1-\lambda_{\mathrm{f}}^{*}\right) \beta+1}{\left(T_{\mathrm{f}}^{*}+\lambda_{\mathrm{f}}^{*}\right) \beta},
$$

where $\beta=\frac{k \kappa T}{q(1-R)} ; \lambda_{\mathrm{f}}^{*}=\lambda_{\mathrm{f}} / C T$, and $T_{\mathrm{f}}^{*}=T_{\mathrm{f}} / T$ are dimensionless values; $k$, the laser-radiation absorption coefficient; $C$, heat capacity; $T$, surface temperature; $T_{\mathrm{f}}$, melting point; and $\lambda_{\mathrm{f}}$, heat of fusion. Numerical estimates showed that the melt layer thickness was less than $5 \cdot 10^{-8} \mathrm{~m}$, i.e., equal to half the layer vaporized by the DLP for defocused radiation $(f= \pm 10 \mathrm{~mm})$.

The amount of vaporized substance decreased and the spectral line intensities weakened for laser ablation of the substance by defocused radiation $\left(q=2.5 \cdot 10^{7} \mathrm{~W} / \mathrm{cm}^{2}\right)$. Therefore, the strongest lines of $\mathrm{Sn}$ and $\mathrm{Cu}$ had to be used as the analytical lines. The reduced amount of vaporized substance caused the particle density in the plume to decrease. Therefore, self-absorption of $\mathrm{Cu}$ could be neglected and the resonance spectral lines (324.754 and $327.396 \mathrm{~nm})$ could be used.

It was advisable to record spectra at a minimum of 10 points and to use the summed spectral line intensity over all points after the $i$-th pulse in order to minimize the uncertainty of layer-by-layer analysis of the coatings. Socalled selective vaporization related to the different melting points of $\mathrm{Sn}\left(232^{\circ} \mathrm{C}\right)$ and $\mathrm{Cu}\left(1083^{\circ} \mathrm{C}\right)$ also occurred for $q=2.5 \cdot 10^{7} \mathrm{~W} / \mathrm{cm}^{2}$. Therefore, Sn would be preferentially vaporized at the start of the pulse so that the laser radiation would in later stages affect a surface where the $\mathrm{Cu}$ concentration was greater than in the sample. Selective vaporization could be neglected for high flux densities. A calibration curve for $\mathrm{Sn}$ was constructed in standard coordinates for layer-by-layer quantitative analysis of the bronze coatings:

$$
I_{\mathrm{Sn}}=k_{1} C_{\mathrm{Sn}}+k_{2}
$$

the $R^{2}$ was 0.92 .

Figure 4 shows the element concentration distributions with depth of bronze coating of steel bead wire. The iron concentration was determined using the following formula:

$$
C_{\mathrm{Fe}}(\%)=100 \%-C_{\mathrm{Cu}}(\%)=C_{\mathrm{Sn}}(\%) .
$$


The increasing Fe concentration with depth of the bronze coating was due to the fact that the steel wire was not polished before applying the coating. Therefore, it contained iron "studs", the size of which increased with layer depth. The magnitude of the irregularities decreased during subsequent drawing so that the coating became more even.

Conclusion. Processes for entry of bronze alloys into laser-induced plasma at various DLP radiation flux densities were examined. It was found that $\mathrm{Sn}$ affected $\mathrm{Cu}$ vaporization by laser radiation for $q=10^{10} \mathrm{~W} / \mathrm{cm}^{2}$. Therefore, the calibration curve for $\mathrm{Cu}$ had to be constructed in relative coordinates. Calibration curves for the other bronze components $(\mathrm{Zn}, \mathrm{Pb}$, and $\mathrm{Sn})$ that were constructed in standard coordinates were approximated well by linear functions.

DLP LIBS was the preferred method for layer-by-layer quantitative analysis of micron-thin bronze coatings because it could determine the element concentrations without preliminary chemical and mechanical surface conditioning and provided a constant layer thickness. It was advisable to use a laser beam that was defocused relative to the surface in order to reduce the power density because the use of light filters weakened significantly the analytical signal and, therefore, increased the experimental uncertainty. The surface temperature of the irradiated sample and, consequently, the thickness of the metal melt decreased for laser-radiation power density $\sim 2.5 \cdot 10^{7} \mathrm{~W} / \mathrm{cm}^{2}$. Hence, components of the bronze coating entered the plasma primarily as individual atoms and the role of selective vaporization increased.

\section{REFERENCES}

1. A. W. Miziolek, V. Palleschi, and I. Schechter, eds., Laser-Induced Breakdown Spectroscopy (LIBS): Fundamentals and Applications, Cambridge, Cambridge University Press (2006).

2. D. A. Cremers and L. J. Radziemski, Handbook of Laser-Induced Breakdown Spectroscopy, John Wiley and Sons, New York (2006).

3. R. Noll, H. Bette, A. Brysch, M. Kraushaar, I. Mönch, L. Peter, and V. Sturm, Spectrochim. Acta, Part B, 56, No. 6, 637-649 (2001).

4. W. Lei, V. Motto-Ros, M. Boueri, Q. Ma, D. Zhang, L. Zheng, H. Zeng, and J. Yu, Spectrochim. Acta, Part B, 64, No. 9, 891-898 (2009).

5. V. S. Burakov and S. N. Raikov, Spectrochim. Acta, Part B, 62, No. 3, 217-223 (2007).

6. A. Santagata, A. De Giacomo, O. De Pascale, M. Dell'Aglio, R. Teghil, A. De Bonis, M. Corrente, G. P. Parisi, and S. Orlando, J. Phys.: Conf. Ser., 59, 585-590 (2007).

7. A. De Giacomo, M. Dell'Aglio, F. Colao, R. Fantoni, and V. Lazic, Appl. Surf. Sci., 247, No. 1, 157-162 (2005).

8. L. Fornarini, F. Colao, R. Fantoni, V. Lazic, and V. Spizzichino, Spectrochim. Acta, Part B, 60, No. 3, 11861201 (2005).

9. L. St-Onge and M. Sabsabi, Spectrochim. Acta, Part B, 55, No. 3, 299-308 (2000).

10. G. Nicolas, M. P. Mateo, and A. Yañez, Appl. Surf. Sci., 254, No. 4, 873-878 (2007).

11. E. S. Voropay and K. F. Ermalitskaia, Vestn. Beloruss. Gos. Univ., Ser. 1: Fiz., Mat. Inf., No. 3, 3-6 (2008).

12. Yu. P. Raizer, Laser Spark and Propagation of Discharges [in Russian], Nauka, Moscow (1974).

13. J. A. Aguilera, C. Aragón, G. Cristoforetti, and E. Tognoni, Spectrochim. Acta, Part B, 64, No. 7, 685-689 (2009).

14. A. A. Petrov and E. A. Pushkareva, Correlation Spectral Analysis of Substances. Book 2: Condensed Phase Analysis [in Russian], Khimiya, St. Petersburg (1993).

15. L. Caneve, F. Colao, R. Fantoni, and V. Spizzichino, Appl. Phys. A: Mater. Sci. Process., 85, No. 2, 151-157 (2006).

16. K. F. Ermalitskaia, in: Abstracts of Papers from the International Scientific Forum "Lomonosov-2009" [in Russian], April 13-18, 2009, Moscow (2009), 7.

17. J. M. Vadillo and J. J. Laserna, Spectrochim. Acta, Part B, 59, No. 2, 147-161 (2004).

18. L. T. Sukhov, Laser Spectral Analysis [in Russian], Nauka, Novosibirsk (1990).

19. V. P. Veiko and E. A. Shakhno, Collection of Problems in Laser Technologies [in Russian], SPSU ITMO, St. Petersburg (2007). 